\title{
Spinal cord compression as the presenting feature of skeletal fluorosis
}

\author{
Zouhour Gassara ${ }^{1}$, Samar Ben djmeaa ${ }^{1}$, Afef Feki ${ }^{1}$, Mohamed Hedi kallel ${ }^{1}$, Hela Fourati ${ }^{1}$, \\ Rim Akrout ${ }^{1}$, and Sofien Baklouti ${ }^{1}$ \\ ${ }^{1}$ Hedi Chaker Hospital
}

January 5, 2021

\begin{abstract}
Skeletal fluorosis is a rare toxic osteopathy reported as an endemic disease and caused especially by prolonged ingestion of high fluoride concentration. Spinal cord compression primarly by the ossification of the posterior longitudinal ligament and ligamentum flavum, may rarely reveal the disease. Surgical decompression is the treatment of choice.
\end{abstract}

\section{Introduction:}

Skeletal fluorosis is reported as an endemic disease in some parts of the world as the north of Africa where the prevalence varies between 20 and $65 \%$ (1). It is a toxic osteopathy caused especially by prolonged ingestion of water having high fluoride concentration or rarely by inhalation of fluoride ions in geographic areas where high levels of fluoride occur naturally $(2,3)$. SF is often asymptomatic. Most common symptoms of SF are bone pain, muscle weakness, bone deformities and pathologic fractures. Neurological complications are rare and include predominantly radiculo-myelopathies ; the majority of those involve the cervical region (4). We present three cases of SF revealed by spinal cord compression.

\section{Case reports:}

Case 1: A 63-year-old man from a rural region where people regularly consume well water with fluoride dust, presented to our department with quadriplegia and urinary incontinence. His symptoms started insidiously with pain in the joints and the back. Then, he started having weakness of upper and lower limbs with difficulty in walking. Clinical examination revealed stiffness of the neck and back, a spastic quadriplegia with stretch reflex hyperactivity and presence of Babinski sign. Blood tests were normal. On X-rays of cervical and thoracic spine, a diffuse osteocondensation was found. A cervical CT revealed a spinal cord compression by exuberant osteophytosis and ossification of longitudinal ligament at cervical levels (C4-C7). MRI revealed signs of myelopathy with high signal intensity T2 of the spinal cord (Fig.1) . Only symptoms treatment (analgesic and non-steroidal anti-inflammatory drugs) was proposed for this patient. Surgical decompression was discussed but not performed because of the high-risk-surgery.

Case 2: A 70-year-old man from south of Tunisia, presented to our department with 1-year history of weakness of upper and lower limbs with urinary incontinence. Clinical examination revealed a severe spastic quadriparesis, a pyramidal syndrome with a sensory level (T4) accompanied by back pain with limited spinal mobility. In addition, brownish mottling was observed on his teeth. Plain X-rays of cervical and thoracic spine and computed tomography (CT) revealed diffuse osteosclerosis with exuberant osteophytosis and ossification of the posterior longitudinal ligament. In addition, an ossification of interosseous membranes on forearms and legs-X-rays was found(Fig.2) . Biological tests were normal. Magnetic resonance imaging (MRI) revealed a spinal cord compression due to ossification of the ligamentum flavum and posterior longitudinal ligament at the cervical level (C4-C6) with high signal intensity (T2) of the spinal cord. 
Case 3: A 70-year-old-man from a rural area (Sidi Bouzid, Tunisia) presented to our department with 4-months history of chronic weakness of lower limbs with urinary incontinence accompanied by neck and lower back pain. On examination, a spastic quadriparesis with a pyramidal syndrome and Babinski sign were revealed. X-rays showed increased bone density with calcification of ligaments, muscular attachment (on pelvis and spine) and interosseous membranes. MRI revealed a spinal cord compression due to ossification of posterior longitudinal ligament at the cervical level. Decompressive laminectomy was proposed for this patient.

\section{Discussion:}

Chronic endemic fluorosis is a major health problem in some regions of the world, especially in India, Asia, and Africa (5).

SF occurs as an endemic disease in some regions of the south of Tunisia especially rural areas because of the consumption of well water having fluoride more than $4 \mathrm{ppm}$, the poor socio-economic status and the hot climate (6). For our three patients, SF was diagnosed based on epidemiological data (endemic region, consumption of well water having high fluoride concentration) associated with radiographic findings (diffuse osteoncondensation, osteophytosis, ossification of ligaments, interosseous membranes and tendon attachments). The diagnosis of SF can be confirmed by the detection of excess fluorine in the blood, urine, or bone tissue. Blood fluorine values higher than $0.2 \mathrm{mg} / \mathrm{l}$ are associated with a high risk of SF (2).

$\mathrm{SF}$ is often asymptomatic but in the advance stages, it causes crippling deformities and neurological complications. Neurological manifestations of SF are predominantly those of radiculo-myelopathies, present in $10 \%$ and the majority of those involved the cervical region. Higher cerebral function defects or cranial nerve palsies are extremely rare $(7,8)$. Radiculo-myelopathy arise primarily from mechanical compression of the spinal cord and nerve roots from osteophytosis, sclerosed vertebral column and ossified ligaments (8). In our cases, the spine cord compression revealed the chronic fluorosis and the compression was caused by posterior vertebral ligament calcification at the cervical region.

MRI with T2 sequences are useful when there is suspicion neurological complication because they demonstrate not only the ligamentous ossification but the degree of spinal cord compression $(2,9,10)$. Decompressive laminectomy is recommended in selected cases of myelopathy caused by ossification of the ligamentum flavum and/or posterior longitudinal ligament. It is only possible in such of those early cases in which the compression is confined to a limited segment of the vertebral column (8). The surgical intervention must be well discussed, considering the surgical risk. In such instances, surgery is fraught with the possibility of complications, including difficulty in intubation because of the rigid cervical spine and high risk for postoperative respiratory complications because of restricted chest movements (9).

\section{Conclusion:}

$\mathrm{SF}$ is a rare chronic metabolic bone disease that the severity lies in the development of skeletal deformities and neurological complications. No current treatment can be proposed except for prevention and stopping etiological factors of chronic fluorosis. Myelopathy is a serious neurological complication that, despite its rarity, can reveal SF. At the stage of neurological complications, surgical decompression is recommended in recent forms with encouraging results.

\section{Author Contributions:}

ZG: collected clinical data, did literature search, and is corresponding author. SB: helped in writing the manuscript and did final proofreading of the submission.

AF: helped in writing the manuscript.

MK, HF, RA and SB: revised and approved the final version of the manuscript.

Acknowledgments: Published with written consent of the patient.

Conflict of interest: No conflict of interest. 


\section{References :}

1. Laatar A, Mrabet D, Zakraoui L. Fluorosis in Sub-Saharan Africa. Revue du Rhumatisme. 2003;70.

2. Sellami M, Riahi H, Maatallah K, Ferjani H, Bouaziz MC, Ladeb MF. Skeletal fluorosis: don't miss the diagnosis! Skeletal Radiol. 2020;49(3):345-357.

3. Datta P, Datta PP. PREVALENCE, ETIOLOGY AND CLINICAL FEATURES OF SKELETAL FLUOROSIS: A CRITICAL REVIEW. Innovare Journal of Medical Sciences. 2013;5-6.

4. Haimanot RT. Neurological complications of endemic skeletal fluorosis, with special emphasis on radiculomyelopathy. Paraplegia. 1990;28(4):244-251.

5. Kurdi MS. Chronic fluorosis: The disease and its anaesthetic implications. Indian J Anaesth. 2016;60(3):157-162.

6. Hafsa C, Zaghouani H, Kriaa S, Salem R, Jerbi-Omezzine S, Golli M, et al. Compression médullaire révélant une fluorose osseuse. Revue du Rhumatisme. 2008;75(1):97-99.

7. Gupta AK, Singh TP, Agrawal PK, Singh D, Sachan M, Agarwal V. Quadriparesis - A rare presentation of skeletal fluorosis. Journal, Indian Academy of Clinical Medicine. 2008;9:201-204.

8. Reddy DR. Neurology of endemic skeletal fluorosis. Neurol India. 2009;57(1):7-12.

9. Geber J, Hammer N. Ossification of the Ligamentum Flavum in a Nineteenth-Century Skeletal Population Sample from Ireland: Using Bioarchaeology to Reveal a Neglected Spine Pathology. Sci Rep. 2018;8(1):9313.

10. Muthukumar N. Ossification of the ligamentum flavum as a result of fluorosis causing myelopathy: report of two cases. Neurosurgery. 2005;56(3).

Figure legends:

Figure 1 : Skeletal fluorosis. MRI of cervical spine: ossification of the posterior longitudinal ligament with compression and high signal intensity of the spinal cord.

Figure 2: Skeletal fluorosis. X-rays of forearms: ossification of interosseous membranes. 


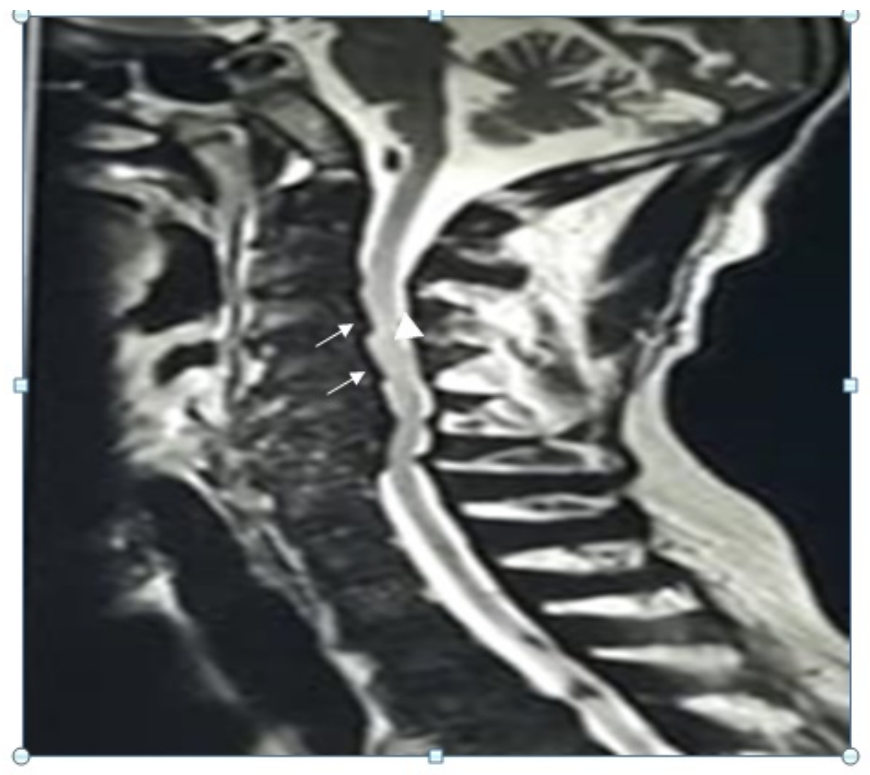

Fig.1: Skeletal fluorosis. MRI of cervical spine (T2): ossification of the posterior longitudinal ligament (white arrows) with compression and high signal intensity of the spinal cord (white arrowhead

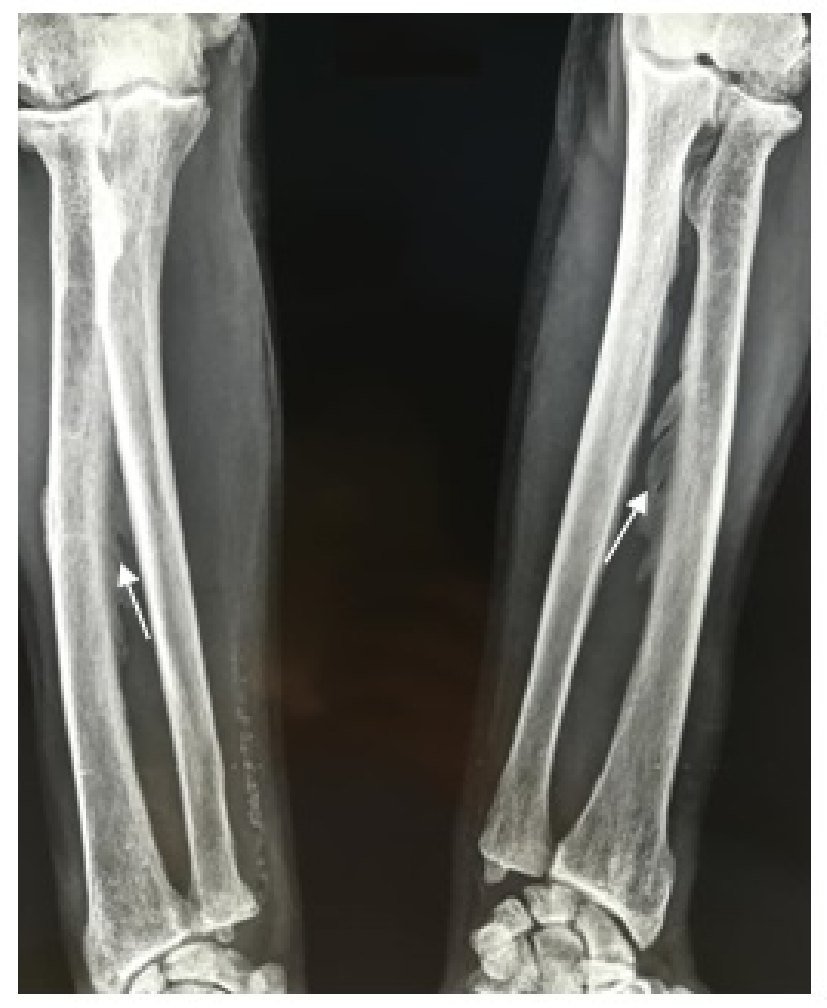

Fig.2: Skeletal fluorosis. X-Rays of forearms: Ossification Of interosseous membranes (arrows). 